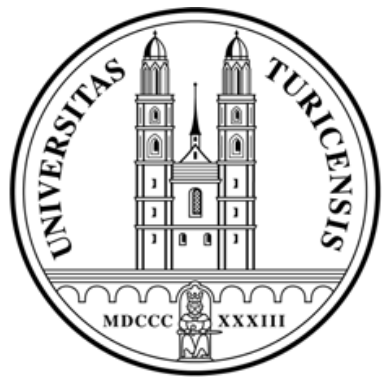

Institute for Empirical Research in Economics

University of Zurich

Working Paper Series

ISSN 1424-0459

Working Paper No. 188

Existence of Sunspot Equilibria and

Uniqueness of Spot Market Equilibria:

The Case of Intrinsically Complete Markets

Thorsten Hens, Janos Mayer and Beate Pilgrim

May 2004 


\title{
Existence of Sunspot Equilibria and Uniqueness of Spot Market Equilibria: The Case of Intrinsically Complete Markets
}

\author{
Thorsten Hens ${ }^{1}$, Janós Mayer ${ }^{2}$ and Beate Pilgrim ${ }^{3}$ \\ 1 IEW, Department of Economics, University of Zurich, Switzerland \\ thens@iew.unizh.ch \\ 2 IOR, Department of Economics, University of Zurich, Switzerland \\ ${ }^{3}$ Reuters AG, Frankfurt, Germany
}

Summary: We consider economies with additively separable utility functions and give conditions for the two-agents case under which the existence of sunspot equilibria is equivalent to the occurrence of the transfer paradox. This equivalence enables us to show that sunspots cannot matter if the initial economy has a unique spot market equilibrium and there are only two commodities or if the economy has a unique equilibrium for all distributions of endowments induced by asset trade. For more than two agents the equivalence breaks and we give an example for sunspot equilibria even though the economy has a unique equilibrium for all distributions of endowments induced by asset trade.

\section{Introduction}

The purpose of this paper is to clarify the relation between the existence of sunspot equilibria and the uniqueness of spot market equilibria for economies with intrinsically complete asset markets. In these economies Pareto-efficient allocations can be attained as competitive equilibria even without asset trade.

[5] introduce their famous paper "Do sunspots matter?" with the question: "What is the best strategy for playing the stock market? Should one concentrate on fundamentals or should one instead focus on the psychology of

\footnotetext{
${ }^{3}$ We would like to thank Anke Gerber, Piero Gottardi, Andreu Mas-Colell and Mike Jerison for very fruitful discussions. Last but not least we are grateful to Klaus Schenk-Hoppé for his support in using $M A T L A B^{\circledR}$ and to Andreas Tupak for his help with the manuscript. All remaining errors are ours.
} 
the market". The sunspot literature, originating from [4] contributes to this question by emphasizing that even if all market participants are completely rational (in the sense that they are maximizing expected utility functions and have rational expectations), still market outcomes can depend on the psychology of the market. The latter is modelled in the sunspot literature as a publicly observed exogenous random event, nicknamed "sunspot". Hence, even without referring to any kind of bounded rationality the best way of playing the stock market will not only be based on fundamentals! The simplest case in which this reasoning can be shown to be correct arises when the economic fundamentals allow for multiple equilibria. The sunspot is then used as a coordination device. In this case sunspot equilibria are similar to correlated equilibria studied in the game theory literature. The point of this paper is to analyze whether this is all sunspot equilibria are about, i.e. whether sunspots matter even when spot market equilibria are unique. Throughout the paper we assume that agents are strictly risk averse. Hence if utilities differ across sunspot states then sunspots matter because sunspot equilibria are Pareto-inefficient (cf. [5]).

We show that for the case of two agents, with utility functions being concave transformations of additively separable functions, the existence of sunspot equilibria is equivalent to the occurrence of the transfer paradox. As an application of this equivalence we demonstrate that the transfer paradox can occur even if the economic fundamentals can be represented by a representative consumer, which in particular implies the uniqueness of equilibria in the initial economy. We also show that the occurrence of sunspot equilibria is indeed subject to the same critique as the occurrence of the transfer paradox. In a model with two commodities, sunspot equilibria can only occur if the initial equilibrium (the equilibrium without asset trade) is not unique. Moreover, if as in the case of Cobb-Douglas economies, uniqueness of equilibria is guaranteed for (almost) all distributions of endowments then sunspot equilibria cannot occur at all. Then we show, again using the equivalence between the two paradoxes, that nevertheless the occurrence of sunspot equilibria does not need to be based on the exogenous selection among multiple equilibria. We construct a simple example in which the equilibrium of any sunspot state is not an equilibrium of any other sunspot state. This example is based on the idea that financial markets may specify incomplete insurance against the uncertainty that they induce. That is to say, in this example asset payoffs are sunspot-dependent and incomplete.

Constructing explicit numerical examples in general equilibrium models is usually done for a class of economies with a simple enough structure so that excess demand functions remain manageable. Computable general equilibrium models (cf. [35]), examples for the occurrence of the transfer paradox ([27, 6, $7,34,14]$, etc), and examples for multiple equilibria $([24,25,20,21])$ therefore use the class of CES-utility functions. The class of utility functions assumed in this paper (monotonic transformations of additively separable functions) includes CES-functions. Keeping this assumption we broaden our analysis by 
looking into the case of more than two agents (countries). We find that the occurrence of the transfer paradox is then no longer sufficient for the existence of sunspot equilibria. Moreover, we give an example with Cobb-Douglas utility functions and sunspot-dependent assets in which sunspots matter even though there are unique equilibria for all distributions of endowments the economy can arrive at using asset trade.

To get the intuition for our results, note that a sunspot equilibrium consists of a set of spot market equilibria (one for each sunspot state) where the endowments of each spot market economy are obtained by redistributions resulting from the equilibrium asset market allocation. Hence an unfavorable redistribution of endowments as it occurs exogenously in the transfer paradox can be derived from asset trade only if the resulting income transfers can be made compatible with the asset allocation the agents choose. In the asset market equilibrium the sum across states (adjusted by the common probability beliefs) of the products of marginal utilities and wealth transfers has to be equal to zero for all agents. Since marginal utilities are non-negative, this requires first of all that all agents find some state with positive income transfer and some with negative income transfer arising from asset trade. Moreover, supposing that marginal utilities are inversely ordered than utility levels, this requires that for some state in which an agent receives a negative income transfer he has a higher utility than for some state with a non-negative income transfer. This is achieved by the transfer paradox. In a sense the transfer paradox then "crosses the order" of marginal utility levels across sunspot states. Note that otherwise all states with negative income transfers would be weighted higher than those with positive income transfer and the probability adjusted sum of marginal utilities times income transfers cannot equalize zero. Hence, provided marginal utilities are inversely related to utility levels the transfer paradox is necessary for sunspots to matter. Moreover, by appropriate choice of the agents' risk aversions we can also show the converse, i.e. that a slightly stronger version of the transfer paradox is indeed sufficient for sunspots to matter. The role of assuming concave transformations of additive separable utilities in this reasoning is to guarantee that indeed higher marginal utilities are implied by lower utility levels. This relation is neither true without concavity of the transformation, without additive separability or with more than two agents. In particular, with more than two agents, the existence of sunspot equilibria is no longer tied to the occurrence of the transfer paradox. Of course, a rigorous argument for this intuition will be given once we have made precise the setup of the model considered.

The question concerning the relation of the existence of sunspot equilibria and the uniqueness of spot market equilibria has also been addressed by [29] and [16], for example. One of our results shows that the construction suggested by [29] can indeed be used to show that, using asset trade, an economy can arrive at an endowment distribution for which there are multiple equilibria even though for the initial distribution of endowments equilibria are unique. Note that in [29] it is not shown that "trading towards multiplicity" can be 
done by starting at an initial distribution of endowments with a unique spot market equilibrium because it is not shown that the equilibrium of the initial economy is unique. Also the Cobb-Douglas example in which sunspot equilibria exist even though spot market equilibria are unique (for all distributions one can arrive at using asset trade) clarifies a confusion that has recently come up in [19] and [3]. [19] has claimed that such an example is possible, but [3] have shown that for the particular parameter values [19] has chosen this is not true! Note that the sunspot equilibrium [3] suggest to consider instead of [19]'s example, sunspots do not matter because agents are not risk averse. Our results in this paper show that the problem in [19] has nothing to do with the parameter values chosen. It arises because [19] considered an economy with only two agents. As we show here, with three agents the construction in [19] is actually possible.

Finally, note that the relation of sunspot equilibria and uniqueness of spot market equilibria has also been studied in economies in which a Paretoefficient allocation cannot be obtained without asset trade. These types of economies originate in the famous "leading example" of [4]. With first period consumption, asset trade may occur because of intertemporal substitution and as a by-product this may introduce extrinsic uncertainty as [4] has first pointed out.

[16] study an intrinsically incomplete economy a la [4], however with real instead of nominal assets. In our paper assets also have real payoffs but the result of [16], who show the existence of sunspot equilibria for a strong uniqueness assumptions on the underlying economy, does not apply to our setting because it relies on first period consumption. Also for the same reason the technique developed by [16] is not applicable here because they control the agents utility gradients both by perturbing the utility functions and by changing the level of first-period consumption.

In the next section we outline the model. Then we give the definitions of the transfer paradox and of sunspot equilibria. Thereafter we prove our result establishing the equivalence of the transfer paradox and the occurrence of sunspot equilibria. Section 4 applies this result to derive some new insights both for sunspot equilibria and also for the transfer paradox. Also in Section 4 we also show some implications for the existence of sunspot equilibria when spot market equilibria are unique. Section 5 concludes.

\section{Model}

We first outline the sunspot model. The transfer paradox will then be embedded into this model by a new interpretation of the sunspot states.

There are two periods. In the second period, one of $s=1, \ldots, S$ states of the world occurs. In the first period assets are traded. Consumption only takes place in the second period. This assumption is important here because 
otherwise the sunspot model cannot be linked to the atemporal transfer paradox model. There are $i=1, \ldots, I$ agents and $l=1, \ldots, L$ commodities in each state. States are called sunspot states because the agents' characteristics within the states, i.e. the agents' endowments $\omega^{i} \in X^{i}$ and their utility functions $u^{i}: X^{i} \rightarrow \mathbb{R}$, do not depend on them. $X^{i}$ is a closed convex subset of $\mathbb{R}_{+}^{L}$ which denotes agent $i$ 's consumption set. In the sunspot literature the agents' characteristics $\left[\left(u^{i}, \omega^{i}\right)_{i=1, \ldots, I}\right]$ are called the economic fundamentals. Throughout this paper we make the

Assumption 1 (Additive Separability)All agents' von Neumann-Morgenstern utility functions $u^{i}$ are monotonic transformations of additively separable functions, i.e. $u^{i}\left(x_{1}^{i}, \ldots, x_{L}^{i}\right)=f^{i}\left(\sum_{l=1}^{L} g_{l}^{i}\left(x_{l}^{i}\right)\right)$ for all $x^{i} \in X^{i}$, where the functions $f^{i}$ and $g_{l}^{i}, l=1, \ldots, L$ are assumed to be twice continuously differentiable, strictly increasing and the $g_{l}^{i}$ are concave. Moreover, we assume that for every agent $i$ at least $L-1$ of the functions $g_{l}^{i}$ are strictly concave and that for all commodities $l$ there is some $i$ for which $g_{l}^{i}$ is strictly concave. Finally, $u^{i}$, is assumed to be concave.

Note that the assumptions on the functions $g_{l}^{i}$ guarantee strict quasi-concavity of the function $u^{i}$. (Strict-) Concavity of $u^{i}$ however also depends on the monotonic transformation $f^{i}$. The class of utility functions covered by assumption 1 includes all utility functions that are commonly used in applied general equilibrium theory. In particular, the case of CES utilities, $u^{i}\left(x^{i}\right)=\sum_{l=1}^{L}\left(\left(\alpha_{l}^{i}\right)^{1-\rho^{i}}\left(x_{l}^{i}\right)^{\rho^{i}}\right)^{1 / \rho^{i}}$, defined for all $i=1, \ldots, I$ on $X^{i}=$ $\left\{x \in \mathbb{R}_{++}^{L} \mid u^{i}(x) \geq u^{i}\left(\omega^{i}\right)\right\}$, for some $\omega^{i} \in \mathbb{R}_{++}^{L}$ and some $0<\alpha_{l}^{i}<1$, $l=1, \ldots, L$ and $\rho^{i}<1$, is covered by this assumption. Also across states agents are assumed to have additive separable utility functions:

Assumption 2 (Expected Utility)For all agents, $i=1, \ldots, I$, the expected utility functions, defining preferences over state contingent consumption $x^{i}(s) \in \mathbb{R}^{L}, s=1, \ldots, S$ are given by

$$
\mathbb{E} u^{i}\left(x^{i}(1), \ldots, x^{i}(S)\right)=\sum_{s=1}^{S} \pi(s) h^{i}\left(u^{i}\left(x^{i}(s)\right)\right) \quad \forall x^{i} \in\left(X^{i}\right)^{S},
$$

where the $h^{i}$ are twice continuously differentiable, strictly increasing and concave functions. Moreover, $\mathbb{E} u^{i}$ is assumed to be strictly concave.

An important subclass of economies arises if the von Neumann-Morgenstern utility functions $h^{i}\left(f^{i}\left(\sum_{l} g_{l}^{i}\left(x_{l}^{i}\right)\right)\right)$ are concave transformations of additively separable functions, i.e. if assumption 1 holds and the composite functions $h^{i} \circ f^{i}$ are concave.

Assumption 3 (E.U. with Concave Additive Separability)Assumption 1 and 2 hold and the composite functions $h^{i} \circ f^{i}$ are concave. 
To include CES-functions under assumption 3, the convex transformation $f^{i}(y)=y^{1 / \rho^{i}}$ has to be transformed by a sufficiently concave function $h^{i}$ so that $h^{i} \circ f^{i}$ is concave. However, to satisfy assumptions 1 and 2 one could choose a strictly concave function that makes the expected utility concave without requiring concavity of $h^{i} \circ f^{i}$. Unfortunately, this subtle difference will be important for our paper, as we will give examples with CES-utilities, satisfying assumptions 1 and 2 but not 3 , in which sunspot equilibria occur.

In the first period agents can trade $j=1, \ldots, J$, real assets with payoffs $A^{j}(s) \in \mathbb{R}^{L}$ if state $s$ occurs. We denote asset prices by $q \in \mathbb{R}^{J}$. Agent $i$ 's portfolio of assets is denoted by $\theta^{i} \in \mathbb{R}^{J}$. Note that all asset payoffs are real, i.e. in terms of commodities. Moreover, we allow for sunspot depended asset payoffs. There is an impressive strand of the sunspot literature originating from [4] in which asset payoffs are nominal. In this literature asset payoffs measured in real terms differ across sunspot states if and only if sunspots matter. The same is effectively also the case in our setting: Supposing spot market equilibria are unique the equilibrium transfers across states measured in real terms depend on sunspots if and only if sunspots matter.

All equilibria we consider in this setting are special cases of competitive equilibria, which are defined in

Definition 1 (Competitive Equilibrium). A competitive equilibrium is an allocation $\left(\stackrel{\star}{x}^{i}, \stackrel{\star}{\theta}{ }^{i}\right), i=1, \ldots, I$, and a price system $(\stackrel{\star}{p}, \stackrel{\star}{q})$ such that

1. For all agents $i=1, \ldots, I$ :

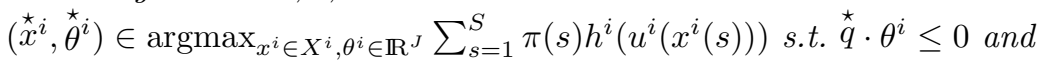

$\stackrel{\star}{p}(s) \cdot x^{i}(s) \leq \stackrel{\star}{p}(s) \cdot \omega^{i}+\stackrel{\star}{p}(s) \cdot A(s) \theta^{i}$ for all $s=1, \ldots, S$.

2. $\sum_{i=1}^{I} \stackrel{\star}{x}^{i}(s)=\sum_{i=1}^{I} \omega^{i}$ for all $s=1, \ldots, S$.

3. $\sum_{i=1}^{I} \stackrel{\star}{\theta}^{i}=0$.

Remark 1. To simplify the exposition when analyzing competitive equilibrium allocations we restrict attention to interior allocations, i.e. to allocations $x^{i}$ in the interior of $X^{i}, i=1, \ldots, I$. A sufficient assumption guaranteeing the interiority of allocations is to impose that the functions $f^{i}$ and $g_{l}^{i}$ satisfy the Inada condition according to which the marginal utility tends to infinity at the boundary of the consumption set $X^{i} \subset \mathbb{R}_{+}^{L}$.

Note that a competitive equilibrium consists of $S$ spot market equilibria (one for each spot market economy with endowments $\left.\hat{\omega}^{i}(s)=\omega^{i}+A(s) \theta^{i}\right)$ together with an asset market equilibrium by which the ex-post endowments of the spot markets are generated. It will be convenient to introduce the spot-market economy of the economic fundamentals as a point of reference. To abbreviate notations we therefore let this economy be the spot market economy in the spot $s=0$. Finally, note that when showing the existence of sunspot equilibria we allow to choose the characteristics not fixed by the economic fundamentals, the sunspot extension, appropriately. The sunspot extension consist of the 
probabilities of the sunspot states $\pi$, the asset structure $A$ and the risk aversion functions $h^{i}$. The sunspot equilibria are robust with respect to perturbations of these characteristics however sunspot equilibria will not exist for all possible choices of the sunspot extension.

This finishes the description of the model.

\section{Sunspot Equilibria and the Transfer Paradox}

In the sunspot literature agents transfer commodity bundles across sunspot states by trading assets. In the international trade literature one thinks of transfers of commodities arising from donations. Each sunspot state will later on be associated with different such donations. The transfer paradox is said to occur if some agent donates some of his resources to some other agent and the recipients utility decreases. In the case of two agents by Pareto-efficiency within spot markets the donor's utility increases. In this statement the utility comparison is done across the competitive equilibria of the economy before and after the donation. In the standard case of the transfer paradox, the transfer was considered to be a transfer of a non-negative amount of commodities $([27])$. In order to make the equivalence to the sunspot model more obvious we consider a slightly more general definition of the transfer paradox which only requires that the donated commodities have non-negative value in the competitive equilibrium after the transfer. As [14] have already shown this generalization is innocuous.

In the following definition we consider alternative possible transfers $\Delta \omega(z)$ that we index by some scenarios $z$. When relating the transfer paradox to sunspot equilibria these scenarios will be associated with different states of the world, $s=1, \ldots, S$. Taking care of potentially multiple equilibria the transfer paradox is then defined as in ${ }^{4}$

Definition 2 (Transfer Paradox). Given an economy with fundamentals $\left[\left(u^{i}, \omega^{i}\right)_{i=1, \ldots, I}\right]$ the transfer paradox occurs if and only if there exists some transfer of endowments $\Delta \omega(z) \in \mathbb{R}^{L I}$, with $\sum_{i=1}^{I} \Delta \omega^{i}(z)=0$ such that for the economy $\left[\left(u^{i}, \omega^{i}+\Delta \omega^{i}(z)\right)_{i=1, \ldots, I}\right]$ there exists an equilibrium $(\stackrel{\star}{x}(z), \stackrel{\star}{p}(z))$ with $\stackrel{\star}{p}(z) \cdot \Delta \omega^{1}(z) \geq 0$ so that $u^{1}\left({ }^{\star} x^{1}(z)\right)<u^{1}\left({ }^{\star} x^{1}(0)\right)$ for some equilibrium $(\stackrel{\star}{x}(0), \stackrel{\star}{p}(0))$ of the economic fundamentals, $\left[\left(u^{i}, \omega^{i}\right)_{i=1, \ldots, I}\right]$, in the reference scenario without transfers, $s=0$.

Note that under certain conditions and if the economic fundamentals have at least two equilibria then even without any transfers the transfer paradox occurs. Our definition covers this case because then $\Delta \omega=0$ is already sufficient to obtain $u^{1}\left(\star^{\star} x^{1}(z)\right)<u^{1}\left(\star^{\star} x^{1}(0)\right)$ for the two equilibria $s=0, z$. Of course if

\footnotetext{
${ }^{4}$ To save on notation we define the transfer paradox with respect to the value of the transfers and changes in utility of agent 1 .
} 
the resulting equilibria are regular then in this case one can also find some transfers of endowments that have positive value and yet the recipients utility decreases. Making the transfer paradox a bit more paradoxical.

We will show that the occurrence of the transfer paradox is a necessary condition for sunspots to matter. To show a converse of this claim we consider the following slightly stronger notion of the transfer paradox.

Definition 3 (Strong Transfer Paradox). Given an economy with fundamentals $\left[\left(u^{i}, \omega^{i}\right)_{i=1, \ldots, I}\right]$ the strong transfer paradox occurs if and only if there exist some transfers of endowments, $\Delta \omega(z) \in \mathbb{R}^{L I}$, with $\sum_{i=1}^{I} \Delta \omega^{i}(z)=0$ and $\Delta \omega(\tilde{s}) \in \mathbb{R}^{L I}$, with $\sum_{i=1}^{I} \Delta \omega^{i}(\tilde{s})=0$ such that for the economies $\left[\left(u^{i}, \omega^{i}+\Delta \omega^{i}(s)\right)_{i=1, \ldots, I}\right], s=z, \tilde{s}$

1. there are some equilibria $(\stackrel{\star}{x}(z), \stackrel{\star}{p}(z)),(\stackrel{\star}{x}(\tilde{s}), \stackrel{\star}{p}(\tilde{s}))$ with $\stackrel{\star}{p}(z) \cdot \Delta \omega^{1}(z) \geq 0$ and $\stackrel{\star}{p}(\tilde{s}) \cdot \Delta \omega^{1}(\tilde{s}) \leq 0$ and

2. it holds that $u^{1}\left(\stackrel{\star}{x}^{1}(z)\right)<u^{1}\left(\stackrel{\star}{x}^{1}(\tilde{s})\right)<u^{1}\left(\stackrel{\star}{x}^{1}(0)\right)$ for some equilibrium $(\stackrel{\star}{x}(0), \stackrel{\star}{p}(0))$ of the economic fundamentals $\left[\left(u^{i}, \omega^{i}\right)_{i=1, \ldots, I}\right]$, in the reference scenario without transfers $s=0$.

Note, that if the economic fundamentals have at least three equilibria then by the same reason as given for the transfer paradox the strong transfer paradox occurs. Hence the existence of at least (three) two equilibria is sufficient for the (strong) transfer paradox. Of course, in regular economies we know that if there are at least two equilibria then there also are at least three equilibria (cf. [9]). This observation indicates that in regular economies the transfer paradox and the strong transfer paradox are actually equivalent. Indeed this it true as the next proposition shows. Recall that in regular economies equilibria are well determined, i.e. in a neighborhood of regular equilibria (being defined by full rank of the Jacobian of market excess demand) there exists a smooth mapping from the exogenous parameters of the economy to the endogenous equilibrium values (cf. [8]). In the following argument regularity needs only be required for the spot market equilibria of the economic fundamentals. This property holds generically in the set of agents' initial endowments $\mathbb{R}_{++}^{L I}$ (cf. [8]).

Proposition 1. Suppose all spot market equilibria of the economic fundamentals $\left[\left(u^{i}, \omega^{i}\right)_{i=1, \ldots, I}\right]$ are regular. Then the transfer paradox and the strong transfer paradox are equivalent.

\section{Proof}

The strong transfer paradox implies the transfer paradox. To establish the converse suppose that the transfer paradox holds. I.e. there exists some transfer of endowments $\Delta \omega(z) \in \mathbb{R}^{L I}$, with $\sum_{i=1}^{I} \Delta \omega^{i}(z)=0$, such that for the economy $\left[\left(u^{i}, \omega^{i}+\Delta \omega^{i}(z)\right)_{i=1, \ldots, i}\right]$ there exists an equilibrium $(\stackrel{\star}{x}(z), \stackrel{\star}{p}(z))$ with $\stackrel{\star}{p}(z) \cdot \Delta \omega^{1}(z) \geq 0$ so that $u^{1}\left(\stackrel{\star}{x}^{1}(z)\right)<u^{1}\left(\stackrel{\star}{x}^{1}(0)\right)$ for some equilibrium $\left(\stackrel{\star}{x}^{i}(0), \stackrel{\star}{p}(0)\right)$ of the economic fundamentals, $s=0$. 
We need to show that there also exists some $\Delta \omega(\tilde{s}) \in \mathbb{R}^{L I}$, with $\sum_{i=1}^{I} \Delta \omega^{i}(\tilde{s})=0$ such that $\stackrel{\star}{p}(\tilde{s}) \cdot \Delta \omega^{1}(\tilde{s}) \leq 0$ and $u^{1}\left(\stackrel{\star}{x}^{1}(z)\right)<u^{1}\left(\stackrel{\star}{x^{1}}(\tilde{s})\right)<$ $u^{1}\left(\stackrel{\star}{x}{ }^{1}(0)\right)$.

This is of course the intuitive case in which a negatively valued transfer leads to a loss in utility. However, we need to ensure that this is the outcome in the spot market equilibrium after the transfer and that the utility loss is not too severe as compared to the loss in the transfer paradox case. This is ensured by the regularity of the equilibrium of the economic fundamentals from which we construct the transfer appropriately: Consider the utility gradient of agent 1, $\nabla u^{1}\left({ }^{\star}{ }^{1}(0)\right)$ at the equilibrium of the economic fundamentals. Choose the transfers $\left(\Delta \omega^{1}(\tilde{s})\right)$, such that $\nabla u^{1}\left({ }^{\star} x^{1}(0)\right)\left(\Delta \omega^{1}(\tilde{s})\right)<0$. By the first order condition of utility maximization in the reference situation $s=0$ we get that this wealth transfer evaluated at the pre-transfer prices is negative, $\stackrel{\star}{p}(0)$. $(\Delta \omega(\tilde{s}))<0$. Since $\nabla u^{1}\left(\stackrel{\star}{x}^{1}(0)\right)(\Delta \omega(\tilde{s}))<0$, by proposition $31.2(i i)$ in [28] we can find some $1 \geq \alpha>0$ such that $u^{1}\left(\stackrel{\star}{x}^{1}(0)+\alpha(\Delta \omega(\tilde{s}))\right)<u^{1}\left(\stackrel{\star}{x}^{1}(0)\right)$. Moreover, by the regularity of the economy, $\alpha>0$ can be chosen small enough so that also the utility at the induced equilibrium is smaller than in the reference situation without transfers, $u^{1}\left({ }^{\star} x^{1}(\tilde{s})\right)<u^{1}\left({ }^{\star} x^{1}(0)\right)$. This is because in regular economies the induced change in the equilibrium allocation $\stackrel{\star}{x}^{1}(\tilde{s})$ can be held small so that $\left|u^{1}\left(\stackrel{\star}{x}^{1}(\tilde{s})\right)-u^{1}\left({ }^{\star} x^{1}(0)+\alpha \Delta \omega(\tilde{s})\right)\right|$ is also small. Moreover, by the same continuity argument this can be done such that $\Delta \omega^{1}(\tilde{s})$ evaluated at prices after the transfer is non-positive, i.e. $\stackrel{\star}{p}(\tilde{s}) \cdot \Delta \omega^{1}(\tilde{s}) \leq 0$. Finally, all this can be done without decreasing the utility level too much, so that for agent 1 we get the inequality $u^{1}\left(\stackrel{\star}{x}^{1}(z)\right)<u^{1}\left(\stackrel{\star}{x}^{1}(\tilde{s})\right)<u^{1}\left(\stackrel{\star}{x}^{1}(0)\right)$.

The strong transfer paradox ensures the order crossing property mentioned in the introduction. To see this note that it is always possible to find transfers of resources, say $\Delta \omega(\hat{s})$, such that the transfer to agent 1 has negative value in the resulting equilibrium, i.e. $\stackrel{\star}{p}(\hat{s}) \cdot \Delta \omega^{1}(\hat{s}) \leq 0$, and agent 1 gets a level of utility that is smaller than any of the utility levels considered in the definition of the strong transfer paradox, i.e. $u^{1}\left(\stackrel{\star}{x}^{1}(\hat{s})\right)<u^{1}\left({ }^{\star} x^{1}(z)\right)<u^{1}\left(\stackrel{\star}{x}^{1}(\tilde{s})\right)<u^{1}\left({ }^{\star} x^{1}(0)\right) .^{5}$ By this observation we get three transfers, two with negative value and one with positive value so that the utility decreases for all transfers. As we will see, by assumption 1 , in the case of two agents, we then get that the order of the marginal utilities does not coincide with the order or the reverse order of the

\footnotetext{
${ }^{5}$ Note that these losses in utility as compared to the equilibrium of the economic fundamentals do not conflict with the fact that trade is voluntary because it may well be that the utility of agent 1 derived from his initial endowments is even smaller than the expected utility obtained in the spot market equilibria. Also the agent is assumed to be a price taker, i.e. he cannot enforce the equilibrium of the economic fundamentals.
} 
transfer values, i.e. the order crossing property is also obtained for marginal utilities.

Note that the transfer paradox concerns the ordering of income transfers and utility levels. In the first order condition for asset demand however marginal utilities and not utility levels themselves play a role. Hence we need to know how the levels of marginal utility are related to the utility levels. Keeping prices fixed across different states, by concavity of the utility function, marginal utilities are inversely related to utility levels. This feature occurs for example if the agents have identical and homothetic preferences. In this case however neither sunspot equilibria matter nor the transfer paradox occurs. In general, changes in relative prices induced by redistributions of income are decisive to determine both the level of utility and of marginal utility. It is these changes from which the transfer paradox and also the existence of sunspot equilibria are derived. Nevertheless, with two agents, whose utilities are concave transformations of additively separable functions, we show that marginal utilities are negatively associated to the level of utilities. To make these ideas precise, we first define the agents' indirect utility function and their marginal utility of income within each state without considering the monotonic transformations $h^{i}$ :

Let

$$
v^{i}(s)=v^{i}\left(p(s), b^{i}(s)\right)=\max _{x^{i} \in X^{i}} f^{i}\left(\sum_{l=1}^{L} g_{l}^{i}\left(x_{l}^{i}(s)\right)\right) \quad \text { s.t. } \quad p(s) \cdot x^{i}(s) \leq b^{i}(s)
$$

be the indirect utility of agents $i$ in state $s$. Since the functions $g_{l}^{i}, l=1, \ldots, L$ are concave and since at least $L-1$ of them are strictly concave there is a unique point $x^{i}$ at which the utility attains its maximum, given that for all commodities the prices $p_{l}(s), l=1, \ldots, L$ and the income $b^{i}(s)$ are positive. In our model the income $b^{i}(s)$ will be given by $p(s) \cdot\left(\omega^{i}+A(s) \cdot \theta^{i}\right)$. I.e., the values of the transfers are given by $r^{i}(s)=p(s) \cdot A(s) \cdot \theta^{i}$. In the analysis of the sunspot model the agents' marginal utility of income will be important

$$
\lambda^{i}(s)=\partial_{v} h^{i}\left(v^{i}(s)\right) \partial_{b} v^{i}\left(p(s), b^{i}(s)\right) .
$$

Hence the marginal utilities that determine the asset allocation are given by the marginal utilities within each state, $\partial_{b} v^{i}\left(p(s), b^{i}(s)\right)$, multiplied by the first derivative of the agents' concave transformations $h^{i}$ determining the agents' risk aversion.

The association between levels of utilities and of marginal utilities is an important link between the transfer paradox and sunspot equilibria which we therefore need to define properly:

Definition 4 (Inverse Association of Utilities and Marginal Utilities). We say that for some agent $i$ the levels of marginal utility are inversely associated to the levels of utility if

$$
v^{i}(1) \geq v^{i}(2) \geq \ldots \geq v^{i}(S)
$$


implies

$$
\lambda^{i}(1) \leq \lambda^{i}(2) \leq \ldots \leq \lambda^{i}(S) .
$$

Moreover, if $v^{i}(\tilde{s})<v^{i}(z)$ for some $\tilde{s}, z \in\{1, \ldots, S\}$ then the corresponding inequality in the marginal utilities of income should also be strict.

This definition puts us in the position to state the equivalence of the occurrence of the transfer paradox and the existence of sunspot equilibria.

Theorem 1 (Equivalence Sunspot Equilibria and Transfer Paradox). Suppose assumption 2 holds and all agents' level of marginal utility are inversely associated to their level of utility. Then

1. the transfer paradox is a necessary condition for sunspots to matter and

2. if there are only two agents then the strong transfer paradox is a sufficient condition for sunspots to matter.

\section{Proof}

1. To link the transfer paradox to the sunspot economy consider

$$
r^{i}(s):=\stackrel{\star}{p}(s) \cdot A(s) \stackrel{\star}{\theta^{i}},
$$

i.e. the transfer of income to agent $i$ as generated by asset trade in some competitive equilibrium.

A necessary condition for optimal portfolio choice is

$$
\sum_{s=1}^{S} \lambda^{i}(s) \pi(s) r^{i}(s)=0, \quad i=1, \ldots, I
$$

which we call the first-order conditions for asset demand. ${ }^{6}$

Now suppose that the transfer paradox does not hold. Then negative transfers $r^{i}(s)<0$ are associated with lower utility levels than positive transfers $r^{i}(s)>0$ are. If moreover marginal utilities are inversely associated to utilities then negative transfers are associated with higher marginal utility levels than positive transfers are. Hence the first order condition for asset demand requires that the probability weighted sum of the absolute values of negative transfers is smaller than the probability weigthed sum of positive transfers:

$$
\sum_{s: r^{i}(s)>0} \pi(s) r^{i}(s)>-\sum_{s: r^{i}(s)<0} \pi(s) r^{i}(s), \quad \text { for all } i=1, . ., I .
$$

This however conflicts with asset market clearing, which implies that income transfers must be balanced:

0 .

${ }^{6}$ This condition follows from $\sum_{s} \lambda^{i}(s) \pi(s) p(s) A(s)=\gamma^{i} q$ together with $q \cdot \theta^{i}=$ 


$$
\sum_{i=1}^{I} r^{i}(s)=0, \quad \text { for all } s=1, \ldots, S .
$$

To see this, multiply equation (2) by $\pi(s)$ and sum those equations over states to obtain:

$$
\sum_{s=1}^{S} \sum_{i=1}^{I} \pi(s) r^{i}(s)=0 .
$$

Rearranging terms this implies

$$
\sum_{i=1}^{I}\left(\sum_{s: r^{i}(s)>0} \pi(s) r^{i}(s)-\sum_{s: r^{i}(s)<0} \pi(s) r^{i}(s)\right)=0
$$

which contradicts equation (1).

2. Suppose the strong transfer paradox occurs, then there exist transfers indexed by $\tilde{s}, z$ such that

$$
r^{1}(z) \geq 0, r^{1}(\tilde{s}) \leq 0 \quad \text { and for some equilibria } v^{1}(z)<v^{1}(\tilde{s})<v^{1}(0)
$$

where $v^{1}(0)$ refers to agent 1's utility in an equilibrium of the spot economy $s=0$. Given the utility functions $u^{1}, u^{2}$ and given the total endowments $\omega^{1}+\omega^{2}$ consider the set of Pareto-efficient allocations as being parameterized by the income transfers $r$.

Now we have to distinguish three cases:

Case 1: If $r^{1}(z)>0$

then we know that $b^{1}(z)>0$ and therefore there exists $r^{1}(\hat{s})<0$ sufficiently small such that for the induced $b^{1}(\hat{s})=\left(b^{1}(z)+r^{1}(\hat{s})\right) \geq 0$ we get $v^{1}(\hat{s})<v^{1}(z)$ for some equilibrium in $\hat{s}$. By thisobservation and the strong transfer paradox we have the order crossing property:

$$
r^{1}(\hat{s}) \leq 0, \quad r^{1}(\tilde{s}) \leq 0, \quad r^{1}(z)>0
$$

while

$$
v^{1}(\hat{s})<v^{1}(z)<v^{1}(\tilde{s})
$$

so that by the negative association of marginal utilities to the level of utilities

$$
\lambda^{1}(\hat{s})>\lambda^{1}(z)>\lambda^{1}(\tilde{s}) .
$$

To construct the sunspot equilibrium consider an economy with the three states $s=\hat{s}, \tilde{s}, z$. In this case the first-order conditions for asset demand become:

$$
\lambda^{i}(\hat{s}) \pi(\hat{s})\left|r^{i}(\hat{s})\right|+\lambda^{i}(\tilde{s}) \pi(\tilde{s})\left|r^{i}(\tilde{s})\right|=\lambda^{i}(z) \pi(z)\left|r^{i}(z)\right|, \quad i=1,2 .
$$

Now choose $\pi(z)<1$ sufficiently large (and accordingly $\pi(\hat{s})>0$ and $\pi(\tilde{s})>0$ sufficiently small) such that 


$$
\pi(\hat{s})\left|r^{i}(\hat{s})\right|+\pi(\tilde{s})\left|r^{i}(\tilde{s})\right|<\pi(z)\left|r^{i}(z)\right| .
$$

Note that $\partial h^{i}$ is any continuous, positive and decreasing function. Recall that, $\lambda^{1}(\tilde{s})<\lambda^{1}(z)$ and that $v^{1}(\hat{s})$ is the smallest utility level in the three states. Hence we can choose $h^{1}$ such that $\lambda^{1}(\hat{s})$ is sufficiently large to solve the first order condition for $i=1$. Analogously it follows that $\lambda^{2}(\hat{s})<\lambda^{2}(z)$ and we can choose $h^{2}$ such that $\lambda^{2}(\tilde{s})$ is sufficiently large to solve the first order condition for $i=2$.

To complete the proof we follow the analogous steps as in [29]. Choose $A \in \mathbb{R}^{3 L \times 2}$ such that

$$
r^{1}(s)=p(s) \cdot\left(A^{1}(s)-A^{2}(s)\right) \quad \text { for } s=\tilde{s}, \hat{s}, z .
$$

Finally, note that

$$
\sum_{s} \lambda^{1}(s) \pi(s) p(s) \cdot A^{1}(s)=\sum_{s} \lambda^{1}(s) \pi(s) p(s) \cdot A^{2}(s)
$$

so that we can choose $q_{1}=q_{2}$. Accordingly we choose $\theta^{1}=(1,-1)$, $\theta^{2}=(-1,1)$ so that $q \cdot \theta^{i}=0, i=1,2$ and $\theta^{1}+\theta^{2}=0$. Since we have chosen an economy with two assets, the first-order conditions for asset trade are equivalent to the conditions $\sum_{s} \lambda^{i}(s) \pi(s) p(s) A(s)=\gamma^{i} q$.

Case 2: If $r^{1}(z)=0$ and $r^{1}(\tilde{s})=0$

then by the strong transfer paradox, even without trading any asset, there is a competitive equilibrium in which sunspots matter.

Case 3: Finally, the case $r^{1}(z)=0$ and $r^{1}(\tilde{s})<0$

is already covered by the reasoning of the first case if one changes the point of view from agent 1 to agent 2 .

Remark 2. Note that in the theorem above part 1 has been shown for the most general statement without evoking any particular assumption on the asset structure $A \in \mathbb{R}^{S L \times J}$. part 2 however is a stronger claim the more the set of asset structures can be restricted. The choice of the asset structure matters in equation (5) of the proof. One way of restricting $A$ is to only consider numeraire assets so that all assets pay off in the same commodity. Allowing for sunspot dependent assets this is a possible choice in the solution of equation (5). If assets are not allowed to depend on the sunspot states then one can still find an asset structure solving equation (5), provided the three price vectors $p(s), s=\tilde{s}, \hat{s}, z$ are linearly independent. The latter then requires to have at least 3 commodities.

To complete this section we first show that under assumption 3 in the case of two agents the order of the marginal utilities of income is inverse to the order of their (indirect) utilities. Hence not only in the trivial case of identical and homothetic preferences we get this property but we also get it for all 
numerical examples with two agents that have so far been considered in the sunspot and in the transfer paradox literature.

Lemma 1. Suppose there are only two agents. Without loss of generality assume that in a competitive equilibrium

$$
v^{1}(1) \leq v^{1}(2) \leq \ldots \leq v^{1}(S) .
$$

Then under assumption 3 it follows that

$$
\lambda^{1}(1) \geq \lambda^{1}(2) \geq \ldots \geq \lambda^{1}(S)
$$

and that

$$
\lambda^{2}(1) \leq \lambda^{2}(2) \leq \ldots \leq \lambda^{2}(S) .
$$

Moreover, if $v^{1}(\tilde{s})<v^{1}(z)$ for some $\tilde{s}, z \in\{1, \ldots, S\}$ then the corresponding inequality in the marginal utilities of income is also strict.

\section{Proof}

Assume that

$$
v^{1}(\tilde{s}) \leq v^{1}(z) \quad\left(\text { resp. that } v^{1}(\tilde{s})<v^{1}(z)\right) \quad \text { for some } \tilde{s}, z \in\{1, \ldots, S\} .
$$

Then, by monotonicity of the utility function, for some commodity, say $k \in$ $\{l, \ldots, L\}$ we must have that

$$
x_{k}^{1}(\tilde{s}) \leq x_{k}^{1}(z) \quad\left(\text { resp. that } x_{k}^{1}(\tilde{s})<x_{k}^{1}(z)\right) .
$$

Moreover, Pareto-efficiency within spot markets implies that for all states $s=1, \ldots, S$ the marginal rates of substitution are equal across agents, i.e.

$$
\frac{\partial g_{m}^{1}\left(x_{m}^{1}(s)\right)}{\partial g_{l}^{1}\left(x_{l}^{1}(s)\right)}=\frac{\partial g_{m}^{2}\left(x_{m}^{2}(s)\right)}{\partial g_{l}^{2}\left(x_{l}^{2}(s)\right)}
$$

for any pair of commodities $(l, m)$. Note that $x_{m}^{2}(s)=\omega_{m}^{1}+\omega_{m}^{2}-x_{m}^{1}(s)$, $s=1, \ldots, S$. Hence if the functions $g_{l}^{i}$ are concave and if for some agent the function $g_{l}^{i}$ is strictly concave then it follows that

$$
x_{l}^{1}(\tilde{s}) \leq x_{l}^{1}(z) \quad\left(\text { resp. that } x_{l}^{1}(\tilde{s})<x_{l}^{1}(z)\right) \quad \text { for all } \quad l=1, \ldots, L .
$$

Without loss of generality assume that $l=n$ is the numeraire in all states $s=1, \ldots, S$, where $n$ is chosen such that $g_{n}^{1}$ is strictly concave. Hence we have shown that

$$
v^{1}(1) \leq v^{1}(2) \leq \ldots \leq v^{1}(S) \quad\left(\text { with } v^{1}(\tilde{s})<v^{1}(z) \quad \text { for some } \tilde{s}, z\right)
$$

implies for the numeraire that

$$
x_{n}^{1}(1) \leq x_{n}^{1}(2) \leq \ldots \leq x_{n}^{1}(S) \quad\left(\text { with } x_{n}^{1}(\tilde{s})<x_{n}^{1}(z) \quad \text { for some } \tilde{s}, z\right) .
$$


From the first order condition to the maximization problem

$$
\max _{x^{i} \in X^{i}} \sum_{l} g_{l}^{1}\left(x_{l}^{1}(s)\right) \quad \text { s.t. } \quad p(s) \cdot x^{1}(s) \leq b^{1}(s)
$$

we get that $\lambda^{1}\left(p(s), b^{1}(s)\right)=\partial_{y}\left(h^{1} \circ f^{1}\right)(y) \partial g_{n}^{1}\left(x_{n}^{1}(s)\right)$ for all $s=1, \ldots, S$. Since $h^{1} \circ f^{1}, g_{n}^{1}$ are (strictly) concave and since $x_{n}^{1}(s)$ and $y^{1}(s)=\sum_{l} g_{l}^{1}\left(x_{l}^{1}(s)\right)$ are increasing (resp. strictly increasing) in $s$ we get that

$$
\lambda^{1}(1) \geq \lambda^{1}(2) \geq \ldots \geq \lambda^{1}(S) \quad\left(\text { resp. that } \lambda^{1}(z)>\lambda^{1}(\tilde{s})\right) .
$$

The claim for $i=2$ follows analogously from the inverse inequalities

$$
x_{l}^{2}(1) \geq x_{l}^{2}(2) \geq \ldots \geq x_{l}^{2}(S) \text { for } l=1, \ldots, L,
$$

and from

$$
v^{2}(1) \geq v^{2}(2) \geq \ldots \geq v^{2}(S),
$$

the latter inequalities being implied by Pareto-efficiency within spot markets.

Before passing to the next section we want to point out that the assumption of additive separability is indeed tight. The inverse association between the levels of marginal utilities and that of utilities, as shown in lemma 1 does not necessarily hold without additive separability. As the following example shows without additive separability one can find that lower utilities are associated with lower marginal utilities. The endowments in this example are supposed to be the ex-post endowments. Hence they are allowed to depend on the sunspot states since the asset payoffs may depend on them.

Remark 3. Consider a two-agent economy with two commodities. The utility functions $\operatorname{are}^{7}$ :

$$
u^{1}\left(x^{1}\right)=\sqrt{x_{1}^{1} x_{2}^{1}}+x_{2}^{1} \text { and } u^{2}\left(x^{2}\right)=\sqrt{x_{1}^{2} x_{2}^{2}}+x_{1}^{2} .
$$

Note that neither of the two utility functions is additively separable but both are strictly monotonically increasing and strictly concave on $\mathbb{R}_{++}^{2}$ and both satisfy the Inada-conditions. Moreover, note that both utility functions are homogenous of degree one implying that both goods are normal. In situation $s=1$ the ex-post endowments are

$$
\hat{\omega}_{1}^{1}(1)=1, \quad \hat{\omega}_{2}^{1}(1)=5 \quad \text { and } \quad \hat{\omega}_{1}^{2}(1)=4, \quad \hat{\omega}_{2}^{2}(1)=2 .
$$

There is a unique equilibrium ${ }^{8}$ with prices $p(1)=(1,0.7125)$. The equilibrium budgets are:

\footnotetext{
${ }^{7}$ The transformations $h^{i} \circ f^{i}$ are assumed to be the identity, which is a strictly increasing concave function.

${ }^{8}$ All values have been rounded to 4 decimal digits. The exact values can be found at the page http://www.iew.unizh.ch/home/hens/sunspot. Uniqueness can be seen from the graph of the excess demand also shown on the webpage.
} 


$$
b^{1}(1)=4.5623 \text { and } b^{2}(1)=5.4249 .
$$

The resulting allocation is:

$$
x_{1}^{1}(1)=0.5380, \quad x_{2}^{1}(1)=5.6485 \quad \text { and } \quad x_{1}^{2}(1)=4.4620, \quad x_{2}^{2}(1)=1.3515 .
$$

The utility levels are:

$$
u^{1}(1)=7.3917 \text { and } u^{2}(1)=6.9177 .
$$

Marginal utilities within state $1,\left(\partial_{b} v^{i}(1)=\frac{v^{i}(1)}{b^{i}(1)}\right)_{i=1,2}$, are:

$$
\partial_{b} v^{1}(1)=1.6202 \text { and } \partial_{b} v^{2}(1)=1.2752 \text {. }
$$

Now consider a second situation $s=2$ with the same total endowments but with a distribution of ex-post endowments as:

$$
\hat{\omega}_{1}^{1}(2)=5, \quad \hat{\omega}_{2}^{1}(2)=5 \quad \text { and } \quad \hat{\omega}_{1}^{2}(2)=0, \quad \hat{\omega}_{2}^{2}(2)=2 .
$$

Again, there is a unique equilibrium, now with prices $p(2)=(1,1.5113)$. The equilibrium budgets are:

$$
b^{1}(2)=12.5563 \quad \text { and } \quad b^{2}(2)=3.0225 .
$$

The resulting allocation is:

$$
x_{1}^{1}(2)=2.3164, \quad x_{2}^{1}(2)=6.7758 \quad \text { and } \quad x_{1}^{2}(2)=2.6836, \quad x_{2}^{2}(2)=0.2242 .
$$

The utility levels are:

$$
u^{1}(2)=10.7375 \text { and } u^{2}(2)=3.4594 .
$$

Marginal utilities within state 2 are:

$$
\partial_{b} v^{1}(2)=0.8552 \text { and } \partial_{b} v^{2}(2)=1.1445 .
$$

Note that the second agent's utility and his marginal utility has decreased is passing from situation 1 to situation 2. Finally, note that we could also have chosen two strictly concave functions $h^{i}$ such that the same ordering still holds for the marginal utilities $\lambda^{i}(s)=\partial_{v} h^{i}\left(v^{i}\right) \partial_{b} v^{i}(s)$.

The next example shows that for more than two agents the strong transfer paradox is no longer sufficient for the existence of sunspot equilibria. The simple reason is that for agent 1 the strong transfer paradox may occur while the two other agents will not find income transfers of opposite sign.

Remark 4. The example to construct the strong transfer paradox is the famous three country example from [6].

There are three agents and two goods. The utility functions are: 


$$
\begin{aligned}
& u^{1}\left(x_{1}^{1}, x_{2}^{1}\right)=\min \left(x_{1}^{1}, 4.0 x_{2}^{1}\right), \\
& u^{2}\left(x_{1}^{2}, x_{2}^{2}\right)=\min \left(x_{1}^{2}, x_{2}^{2}\right), \\
& u^{3}\left(x_{1}^{3}, x_{2}^{3}\right)=\min \left(2.8 x_{1}^{3}, x_{2}^{3}\right) .
\end{aligned}
$$

Note that this example uses Leontief preferences. Hence strictly spoken our assumption 1 is not satisfied. However, these preferences can be attained as a limit case of CES-utility functions. That is to say, perturbing the preferences slightly within the CES-class will establish an example satisfying assumption 1 and if we like to transform it by a sufficiently concave function $h^{i}$ also assumption 3 can be satisfied, as we mentioned above. Moreover, note that both utility functions, $u^{i}$ are homogenous of degree one implying that both goods are normal. Consider the situations $s=0, \tilde{s}, z, \hat{s}$ as required by the strong transfer paradox.

Let the matrix of endowments (for both goods per agent and state), with rows corresponding to states $s=0, \hat{s}, z, \tilde{s}$ and with columns corresponding to agents, be:

$$
\omega=\left(\begin{array}{lll}
(1,1.00) & (2,1.00) & (1,3.00) \\
(1,0.10) & (2,2.40) & (1,2.50) \\
(1,1.10) & (2,1.00) & (1,2.90) \\
(1,0.80) & (2,1.25) & (1,2.95)
\end{array}\right),
$$

In all situations there is a unique equilibrium ${ }^{9}$. The equilibrium price vectors are:

$$
\begin{aligned}
& p(0)=(5.9084,1), \\
& p(\tilde{s})=(4.4892,1), \\
& p(z)=(9.6382,1), \\
& p(\hat{s})=(0.9438,1) .
\end{aligned}
$$

Evaluated at these equilibrium prices the transfers as compared to situation $s=0$ are:

$$
\begin{aligned}
r^{1}(\tilde{s})=-0.2, & r^{2}(\tilde{s})=0.25 \quad \text { and } \quad r^{3}(\tilde{s})=-0.5 \\
r^{1}(z)=0.1, & r^{2}(z)=0.00 \quad \text { and } \quad r^{3}(z)=-0.1 \\
r^{1}(\hat{s})=-0.9, & r^{2}(\hat{s})=1.40 \quad \text { and } \quad r^{3}(\hat{s})=-0.5
\end{aligned}
$$

The equilibrium budgets are:

$$
\begin{array}{lll}
b^{1}(0)=6.9084, & b^{2}(0)=12.8169 \quad \text { and } \quad b^{3}(0)=8.9084, \\
b^{1}(\tilde{s})=5.2892, & b^{2}(\tilde{s})=10.2284 \quad \text { and } & b^{3}(\tilde{s})=7.4392, \\
b^{1}(z)=10.7382, & b^{2}(z)=20.2765 \text { and } \quad b^{3}(z)=12.5382, \\
b^{1}(\hat{s})=1.0438, & b^{2}(\hat{s})=4.2876 \quad \text { and } \quad b^{3}(\hat{s})=3.4438
\end{array}
$$

\footnotetext{
${ }^{9}$ All values have been rounded to 4 decimal digits. The exact values can be found at the page http://www.iew.unizh.ch/home/hens/sunspot. Uniqueness can be seen from the graph of the excess demand function which is displayed at the webpage together with the computations.
} 
The resulting allocations are:

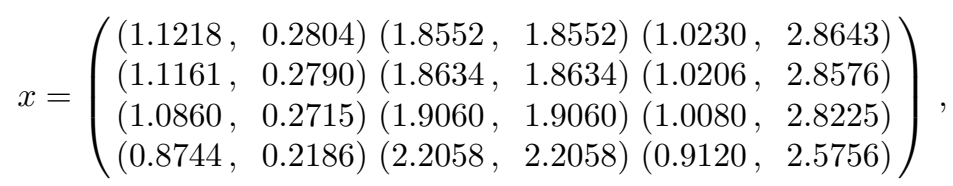

The corresponding utility levels are:

$$
\begin{array}{ll}
u^{1}(0)=1.1218, & u^{2}(0)=1.8552 \quad \text { and } \quad u^{3}(0)=2.8643, \\
u^{1}(\tilde{s})=1.1161, & u^{2}(\tilde{s})=1.8634 \quad \text { and } \quad u^{3}(\tilde{s})=2.8576, \\
u^{1}(z)=1.0860, & u^{2}(z)=1.9060 \quad \text { and } \quad u^{3}(z)=2.8225, \\
u^{1}(\hat{s})=0.8744, & u^{2}(\hat{s})=2.2058 \quad \text { and } \quad u^{3}(\hat{s})=2.5756
\end{array}
$$

Marginal utilities $\left(\partial_{b} v^{i}(s)=\frac{v^{i}(s)}{b^{i}(s)}\right)_{i=1,2,3 \text {. }}$ are:

$$
\begin{aligned}
& \partial_{b} v^{1}(0)=0.1624, \quad \partial_{b} v^{2}(0)=0.1448 \quad \text { and } \quad \partial_{b} v^{3}(0)=0.3215 \\
& \partial_{b} v^{1}(\tilde{s})=0.2110, \quad \partial_{b} v^{2}(\tilde{s})=0.1822 \quad \text { and } \quad \partial_{b} v^{3}(\tilde{s})=0.3841 \\
& \partial_{b} v^{1}(z)=0.1011, \quad \partial_{b} v^{2}(z)=0.0940 \quad \text { and } \quad \partial_{b} v^{3}(z)=0.2251 \\
& \partial_{b} v^{1}(\hat{s})=0.8377, \quad \partial_{b} v^{2}(\hat{s})=0.5145 \quad \text { and } \quad \partial_{b} v^{3}(\hat{s})=0.7479
\end{aligned}
$$

Note that the second agent's transfers are never negative while that of the third agent are never positive. Hence these transfers cannot be sustained by asset trade. Finally, note that as compared to situation $s=0$ the third agent's utility and his marginal utility has decreased in passing to situation $s=z$.

\section{Sunspot Equilibria and Uniqueness of Spot Market Equilibria}

Having established the link between the transfer paradox and the existence of sunspot equilibria we now derive some new results on the existence of sunspot equilibria when spot market equilibria are unique on the one hand and also on the possibility of the transfer paradox on the other hand. Applying part 1 of our theorem and [36] we can rule out sunspot equilibria if marginal utilities are inversely related to utilities and spot market equilibria are unique at all non-negative distributions of endowments. Once again, applying part 1 of our theorem and [2] we can rule out sunspot equilibria in the case of two commodities and two agents if the economic fundamentals do have a unique equilibrium. On the other hand, applying the construction of [29], which is done for the case of two agents, part 2 of our result shows that the transfer paradox can occur even if for the economic fundamentals there exists a representative consumer. We continue, using once more part 2 , to show that sunspot equilibria need not be derived from multiple equilibria of the spot market economy that is obtained by asset trade leading to the same endowment distribution in all states. While [29]'s construction uses that there are 
multiple equilibria for the induced distribution of endowments, this example however exploits the fact that there are multiple equilibria for the initial distribution of endowments. Therefore, we give one more example in which spot market equilibria are unique for all distributions of endowments that can be arrived at by asset trade and still sunspots matter. This final example then settles the question whether sunspot equilibria need to be based on some sort of multiplicity of spot market equilibria.

The following terminology is quite useful. A randomization equilibrium is a competitive equilibrium in which for some ex-post endowments the equilibrium allocation in every state $s$ is a spot market allocation for the same economy. If, for example, the economic fundamentals allow for multiple equilibria then there is a randomization equilibrium. [29] has shown that there can also be randomization equilibria if there are multiple equilibria for some distribution of endowments that is attainable via asset trade. In both cases the equilibrium allocation of such a competitive equilibrium is a randomization among the set of equilibria of some underlying economy. In randomization equilibria sunspots are a device to coordinate agents' expectations. This case of sunspot equilibria has found many applications. In the international trade literature, for example, currency crises are modelled by randomization sunspot equilibria. See, for example, the seminal papers by [31] and [32] and also the interesting empirical papers on this issue by [22] and [23]).

The question that arises from these observations is whether sunspot equilibria could be identified with randomization equilibria. This would then make them very similar to publicly correlated equilibria known in the game theoretic literature $([1])^{10}$. Hence, the results of this literature would then be applicable to sunspot equilibria.

It is obvious that in our setting with intrinsically complete markets sunspot equilibria necessarily are randomization equilibria if assets are sunindependent assets, if $A(s)=A(1), s=1, \ldots, S$. It is, however, not obvious at all whether with a general asset structure there can also be sunspot equilibria which are different from randomization equilibria. To clarify this point some more definitions are needed.

Definition 5 (Attainable Endowment Distributions). Given the economic fundamentals $\left[\left(u^{i}, \omega^{i}\right)_{i=1, \ldots, I}\right]$ and given the asset structure $A$ the endowment distributions $\hat{\omega}^{i}(s), s=1, \ldots, S, i=1, \ldots, I$ is attainable if there exists some competitive equilibrium with asset allocation $\left({ }^{\star}{ }^{i}\right), i=1, \ldots, I$, such that $\hat{\omega}^{i}(s)=\omega^{i}+A(s) \hat{\theta}^{i}, s=1, \ldots, S, i=1, \ldots, I$.

Based on the attainability concept we now define the uniqueness concept suggested in [29]. This condition has later been called no potential multiplicity by $[16]$.

\footnotetext{
${ }^{10}$ See [12] for relating sunspot equilibria to correlated equilibria in an overlapping generations model.
} 
Definition 6 (Strong Uniqueness). The economy with the fundamentals $\left[\left(u^{i}, \omega^{i}\right)_{i=1, \ldots, I}\right]$ satisfies the strong uniqueness property for some asset structure $A$, if the spot market equilibria are unique for every attainable endowment distribution.

Remark 5. With intrinsically incomplete markets sunspots are known to matter even if the economic fundamentals satisfy the strong uniqueness property (cf. [4] and the literature that has emerged from it, [17] and [16]).

As said above, when assets are sun-independent then in our setting with intrinsically complete markets the strong uniqueness property rules out sunspot equilibria. The following corollary to our theorem shows that for general asset structures the strong uniqueness property restricted to non-negative allocations is still sufficient to rule out sunspot equilibria when marginal utilities are inversely related to levels of utility.

Corollary 1. Under assumptions 1 and 2 and if marginal utilities are inversely related to levels of utility then sunspots do not matter if there are unique spot market equilibria for all non-negative distributions of endowments in the Edgeworth box.

\section{Proof}

Suppose sunspots did matter, then from our main result we know that the transfer paradox needs to occur. However, as [36] has shown, this requires to be able to trade to some non-negative distribution of endowments for which there are multiple equilibria, which is a violation of the strong uniqueness property.

Hence applying our lemma 1, in particular in the case of two agents, sunspots do not matter if there are unique spot market equilibria for all distributions of endowments in the Edgeworth box.

However, asking for the strong uniqueness property may be asking too much since not many economic fundamentals will satisfy this property. Hence the question arises whether sunspot equilibria can exist when there are multiple equilibria for some distribution of endowments the economy can arrive at using asset trade. As [29] has shown this is definitely the case. However, [29] did not show that this is still the case if for the initial distribution of endowments there is a unique equilibrium. Note if there were also multiple equilibria at that point then sunspots would matter even without evoking asset trade. The question then is whether "trading from uniqueness to multiplicity" is possible. As the following corollary shows, this is not possible if there are two agents and two commodities.

Corollary 2. Under assumption 3, in the case of two commodities and two consumers sunspots do not matter if the economic fundamentals have a unique equilibrium. 


\section{Proof}

Suppose sunspots did matter then from our main result we know that the transfer paradox needs to occur. However, as for example [2] ${ }^{11}$ has shown, in the case of two commodities this requires to have multiple equilibria for the initial distributions of endowments.

From corollary 2 we can see that in the case of two commodities and sun-independent assets it is not possible to "trade from uniqueness to multiplicity". This is because with sun-independent assets sunspots can only matter at distributions of endowments for which there are multiple equilibria.

[19] has claimed that for an economy with two agents and two commodities in which utility functions are concave transformations of Cobb-Douglas utility functions sunspots matter. The corollary 2 shows that this claim is incorrect. Moreover the mistake in [19] cannot be cured by changing the values of the parameters for the same example ${ }^{12}$ because that example falls into the broad class of economies which are covered by this note. Indeed for CobbDouglas economies the equilibrium at the initial distribution of endowments is unique and the strong uniqueness requirement is satisfied for almost all asset structures $A$.

Making further assumptions on the agents' risk aversion functions $h^{i}$, [33] shows that the construction of [29] does not work if there are only two commodities. Indeed, [33] shows for an economy with an arbitrary number of consumers with additive separable utilities $u^{i}$ and non-decreasing relative risk aversion, with two commodities and with asset payoffs that are independent of the sunspot states, non-trivial sunspot equilibria do not exist.

The next example shows that with more than two commodities [29]'s construction can be followed to demonstrate that it is well possible to trade from uniqueness to multiplicity. Hence sunspot equilibria do occur even if for the initial distribution of endowments there is a unique equilibrium. By our theorem then also the transfer paradox needs to occur because the economy we construct has two consumers with CES-utility functions. That the transfer paradox arises even if the initial equilibrium is unique should be no surprise since this is well known in that literature. In the example we give we do however have some more structure. For the initial distribution of endowments the excess demand function of the economic fundamentals can be generated by a single representative consumer. Hence our example shows that "the representative consumer is not immune against sunspots" and that even under this strong structural assumption the transfer paradox is possible.

\footnotetext{
${ }^{11}$ See also the solution to exercise $15 . \mathrm{B} \cdot 10^{\mathrm{C}}$ from [30] that is given in [18].

${ }^{12}$ This possibility is left open by the observation of [3] who demonstrate that for the specific parameter values chosen in [19] sunspots do not matter!
} 
Corollary 3. There exist economic fundamentals, satisfying assumptions 1 and 2, with two agents for which there is a representative consumer and yet sunspot equilibria and the transfer paradox occur.

\section{Proof}

The example we give to prove this claim follows the construction of [29] making sure that it can be done even under assumption 1 and 2 and more importantly, that it can be done even for economic fundamentals with a representative consumer:

There are four commodities and two CES-consumers with utility functions:

$$
u^{i}\left(x^{i}\right)=\sum_{l=1}^{4}\left(\left(\alpha_{l}^{i}\right)^{1-\rho^{i}}\left(x_{l}^{i}\right)^{\rho^{i}}\right)^{1 / \rho^{i}}
$$

The utility parameters are ${ }^{13}: \rho^{1}=-250.00000, \rho^{2}=-250.00000$ and

$$
\begin{aligned}
& \alpha^{1}=\left(\begin{array}{llll}
0.24986,0.24986,0.25041, & 0.24986)
\end{array}\right) \\
& \alpha^{2}=\left(\begin{array}{llll}
0.25261, & 0.25224, & 0.24758, & 0.24758
\end{array}\right) \text {. }
\end{aligned}
$$

Note that utility functions satisfy assumption 1 and that they are linearly homogenous. Moreover, agents utilities are close to the Leontief case which is convenient because then the Slutzky-subsitution effects contributing to the uniqueness of equilibria are small. Initial endowments are:

$$
\begin{aligned}
& \omega^{1}=(0.01024,0.01021,0.01006,0.0101), \\
& \omega^{2}=(0.01011,0.01009,0.00994,0.01) \text {. }
\end{aligned}
$$

Note that the second agent's initial endowments multiplied with 1.00971 gives the first agent's endowments, i.e. endowments are collinear and hence, by [11]'s theorem, for this economy there exists a representative consumer.

Suppose there are three sunspot states, $s=1,2,3$. We claim that trade in (sun-independent) assets can be used to arrive at the following distribution of endowments for which there are three spot market equilibria:

$$
\begin{aligned}
& \hat{\omega}^{1}=(0.01414,-0.01804,0.01259,0.0197) \text {, } \\
& \hat{\omega}^{2}=(0.00621, \quad 0.03834,0.00741,0.00037) \text {. }
\end{aligned}
$$

The resulting spot market equilibrium prices are:

$$
\begin{aligned}
& p(1)=(1,1.5801, \quad 5.05786,0.88298), \\
& p(2)=(1,1.37985,0.88617,0.35661) \text {, } \\
& p(3)=(1,1.6344,11.79925,3.67601) \text {. }
\end{aligned}
$$

Note that the rank of the price matrix is three since the determinant of all prices excluding the numeraire is -11.11 . The equilibrium budgets are:

\footnotetext{
${ }^{13}$ All values have been rounded to 5 decimal digits. The exact values can be found at the page http://www.iew.unizh.ch/home/hens/sunspot.
} 


$$
\begin{aligned}
& b^{1}(1)=0.06669 \quad \text { and } \quad b^{2}(1)=0.10461, \\
& b^{1}(2)=0.00742 \quad \text { and } \quad b^{2}(2)=0.06582 \text {, } \\
& b^{1}(3)=0.20558 \text { and } b^{2}(3)=0.15770 \text {. }
\end{aligned}
$$

The resulting allocations are:

$$
\begin{aligned}
& x^{1}(1)=\left(\begin{array}{llll}
0.00785, & 0.00783, & 0.00781,0.00785),
\end{array}\right. \\
& x^{2}(1)=(0.01250,0.01246,0.01218,0.01226) \text {, } \\
& x^{1}(2)=\left(\begin{array}{llll}
0.00205, & 0.00204, & 0.00205,0.00206)
\end{array}\right) \\
& x^{2}(2)=\left(\begin{array}{llll}
0.01830 & 0.01825,0.01795,0.01801
\end{array}\right) \text {, } \\
& x^{1}(3)=\left(\begin{array}{llll}
0.01142, & 0.01140, & 0.01134, & 0.01136
\end{array}\right), \\
& x^{2}(3)=\left(\begin{array}{llll}
0.00893, & 0.00890,0.00866,0.00871)
\end{array}\right. \text {. }
\end{aligned}
$$

As was to be expected in a case close to the Leontief-preferences, for both agents and in all states the demand of any good 1 as a fraction of the sum of all demands stay close to the agents utility parameters $\alpha_{l}^{i}$ even though prices vary substantially.

The corresponding utility levels are:

$$
\begin{array}{lll}
u^{1}(1)=0.03132 & \text { and } & u^{2}(1)=0.04935, \\
u^{1}(2)=0.00819 & \text { and } & u^{2}(2)=0.07249, \\
u^{1}(3)=0.04544 & \text { and } & u^{2}(3)=0.03513 .
\end{array}
$$

Marginal utilities $\left(\partial_{b} v^{i}(s)=\frac{u^{i}(s)}{b^{i}(s)}\right)_{i=1,2,3 \text {. }}$ are:

$$
\begin{aligned}
& \partial_{b} v^{1}(1)=0.46960 \quad \text { and } \quad \partial_{b} v^{2}(1)=0.47178, \\
& \partial_{b} v^{1}(2)=1.10458 \quad \text { and } \quad \partial_{b} v^{2}(2)=1.10130, \\
& \partial_{b} v^{1}(3)=0.22104 \quad \text { and } \quad \partial_{b} v^{2}(3)=0.22277 .
\end{aligned}
$$

Note that agent 1's marginal utilities are ordered inversely to his utilities while agent 2's marginal utilities are ordered as his utility levels. This is possible here because, as mentioned above, the assumption 3 is not satisfied for CESutilities. Evaluating the change in endowments at the new equilibrium prices, the resulting income transfers from agent 1's point of view are:

$$
r^{1}(1)=0.00201, \quad r^{1}(2)=0.02195, \quad r^{1}(3)=-0.05989 .
$$

Next we argue that for these values we can solve the first order condition for asset demand:

$$
\sum_{s} h^{i}\left(v^{i}(s)\right) \partial_{b} v^{i}(s) \pi(s) r^{i}(s)=0, i=1,2 .
$$

To do so we can still choose the vector of probabilities $\pi$ and also the degree of the agents' risk aversion given by three values of the function $h^{\prime i}$ which are 
positive and decreasing with $v^{i}$. However, the functions $h^{i}$ are not sufficiently concave to make the composition $h^{i} \circ f^{i}$ concave.

The resulting values are $\pi=(0.8,0.05,0.15)$ for the probabilities and

$h^{\prime 1}=(1.98877,2.00032,1.97707) \quad$ and $\quad h^{\prime 2}=(1.01074,1.00064,1.02074)$

for the risk aversions. Note that these values are smaller the higher the utility levels are.

Since the three spot price vectors are linearly independent, we can now find an asset matrix $A \in \mathbb{R}^{3 \times 4 \times 2}$ such that

$$
r(s)=p(s) \cdot\left(A^{1}(s)-A^{2}(s)\right) \text { for } s=1,2,3 .
$$

The vector $A^{1}-A^{2}$ we have chosen is:

$$
A^{1}-A^{2}=(-0.00597,0.02618,-0.00457,-0.01165) .
$$

Finally, note that

$$
\sum_{s} \lambda^{1}(s) \pi(s) p(s) \cdot A^{1}(s)=\sum_{s} \lambda^{1}(s) \pi(s) p(s) \cdot A^{2}(s)
$$

so that we can choose $q_{1}=q_{2}$. Accordingly we choose $\theta^{1}=(1,-1), \theta^{2}=$ $(-1,1)$ so that $q \cdot \theta^{i}=0, i=1,2$ and $\theta^{1}+\theta^{2}=0$. Since we have chosen an economy with two assets, the first-order conditions for asset trade are equivalent to the conditions $\sum_{s} \lambda^{i}(s) \pi(s) p(s) \cdot A(s)=\gamma^{i} q$.

Comparing the vector of income transfers and the resulting utility levels we find that agents' utility decrease when they receive positive income transfer, i.e. the transfer paradox occurs even though the order crossing property does not.

$$
\left.u^{1}(\text { no-asset-trade })=0.03224 \text { and } \quad u^{2} \text { (no-asset-trade }\right)=0.03242 .
$$

Last but not least, both agents profit from trade, i.e. their reservation utilities are smaller than their utilities when participating in the markets ${ }^{14}$ :

$$
\begin{gathered}
u^{1}(\text { no-trade })=0.03218 \text { and } u^{2}(\text { no-trade })=0.04831 \\
\mathbb{E} u^{1}=0.03228 \text { and } \mathbb{E} u^{2}=0.04838
\end{gathered}
$$

The next corollary shows that as in the case of intrinsically incomplete markets also with intrinsically complete markets sunspots can still matter even if they do not serve as a coordination device among multiple equilibria.

\footnotetext{
${ }^{14}$ In the computation of the expected utility we have ignored the functions $h^{i}$ because they are sufficiently close to the case of risk neutrality. Indeed in this example, the first-order-conditions for asset demand could also be solved without the functions $h^{i}$.
} 
Corollary 4. With sun-dependent assets, under assumption 3, even for the case of two commodities, there are sunspot equilibria which are not randomization equilibria.

\section{Proof}

The example we give to prove this corollary is adapted from the Example 15.B.2 in $[30]^{15}$. There are two commodities and two agents with endowments $\left[\left(\omega_{1}^{1}, \omega_{2}^{1}\right),\left(\omega_{1}^{2}, \omega_{2}^{2}\right)\right]=[(2, r),(r, 2)]$. Consumption sets are $X^{i}=\{x \in$ $\left.\mathbb{R}_{++}^{L} \mid u^{i}(x) \geq u^{i}\left(\omega^{i}\right)\right\}$ and utility functions are given by

$$
u^{1}\left(x^{1}\right)=x_{1}^{1}-\frac{1}{8}\left(x_{2}^{1}\right)^{-8} \quad \text { and } \quad u^{2}\left(x^{2}\right)=-\frac{1}{8}\left(x_{1}^{2}\right)^{-8}+x_{2}^{2} .
$$

Aggregate endowments are $\omega=(2+r, 2+r)$ where $r=2^{\frac{8}{9}}-2^{\frac{1}{9}} \approx 0.77$.

Figure 1 shows the Edgeworth Box of this economy. ${ }^{16}$ The convex curve is

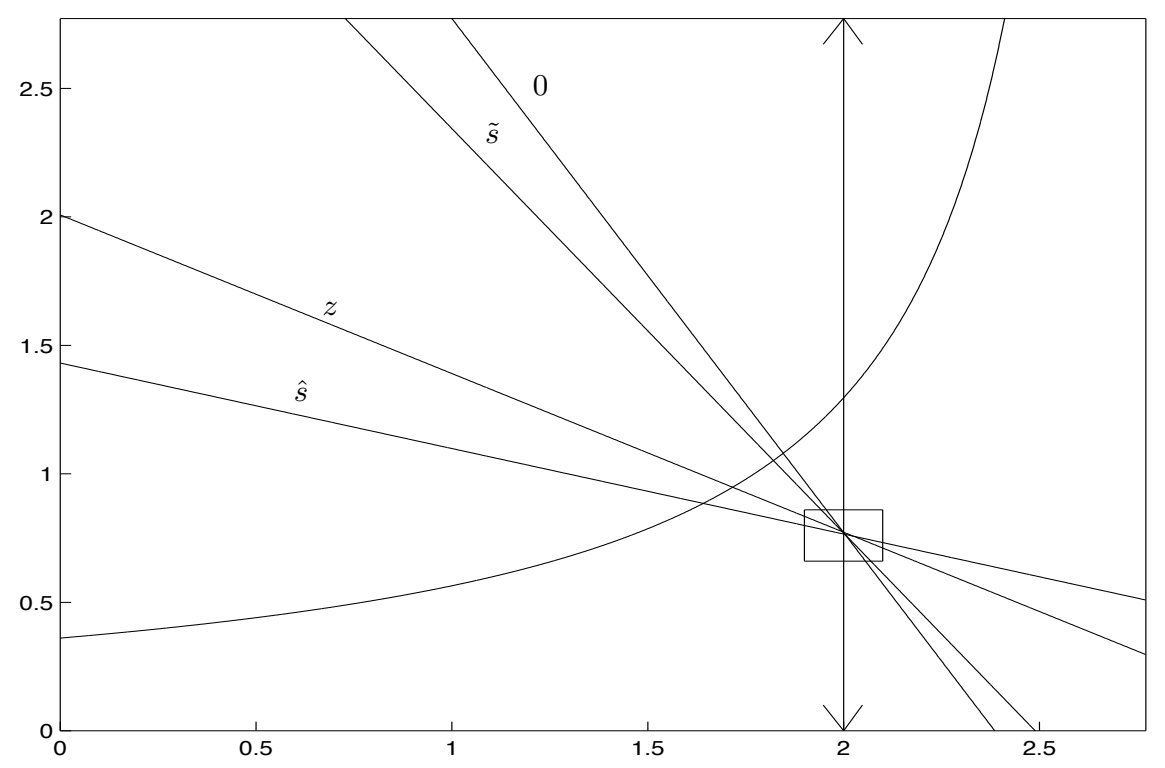

Fig. 1. Edgeworth-Box

the set of Pareto-efficient allocations that lie in the interior of the Edgeworth Box. It is given by the function $x_{2}^{1}=\frac{1}{2+r-x_{1}^{1}}$. The competitive equilibrium allocations of our example will be constructed out of these interior allocations. In figure 1 we have also drawn some budget lines indexed by $s=\hat{s}, z, \tilde{s}, 0$,

\footnotetext{
${ }^{15}$ See [18] for the solution to the original example.

${ }^{16}$ The figures 1 and 2 have been generated with $M A T L A B{ }^{\circledR}$. The scripts can be downloaded from the page http://www.iew. unizh.ch/home/hens.
} 
supporting four different Pareto-efficient allocations which are equilibrium allocations in the spot markets once appropriate spot market endowments have been chosen. The sunspot equilibrium we construct exploits the fact that in this example there are three equilibria for the distribution of endowments $\left[\left(\omega_{1}^{1}, \omega_{2}^{1}\right),\left(\omega_{1}^{2}, \omega_{2}^{2}\right)\right]=[(2, r),(r, 2)]$. Taking these endowments as the reference point for the economy $s=0$, we consider the transfer of endowments as visualized in figure 2 . From the three equilibria at $[(2, r),(r, 2)]$ we have chosen the one with the highest first agent utility to be the equilibrium allocation for the reference situation $s=0$. The asset structure $A$ consists of numeraire

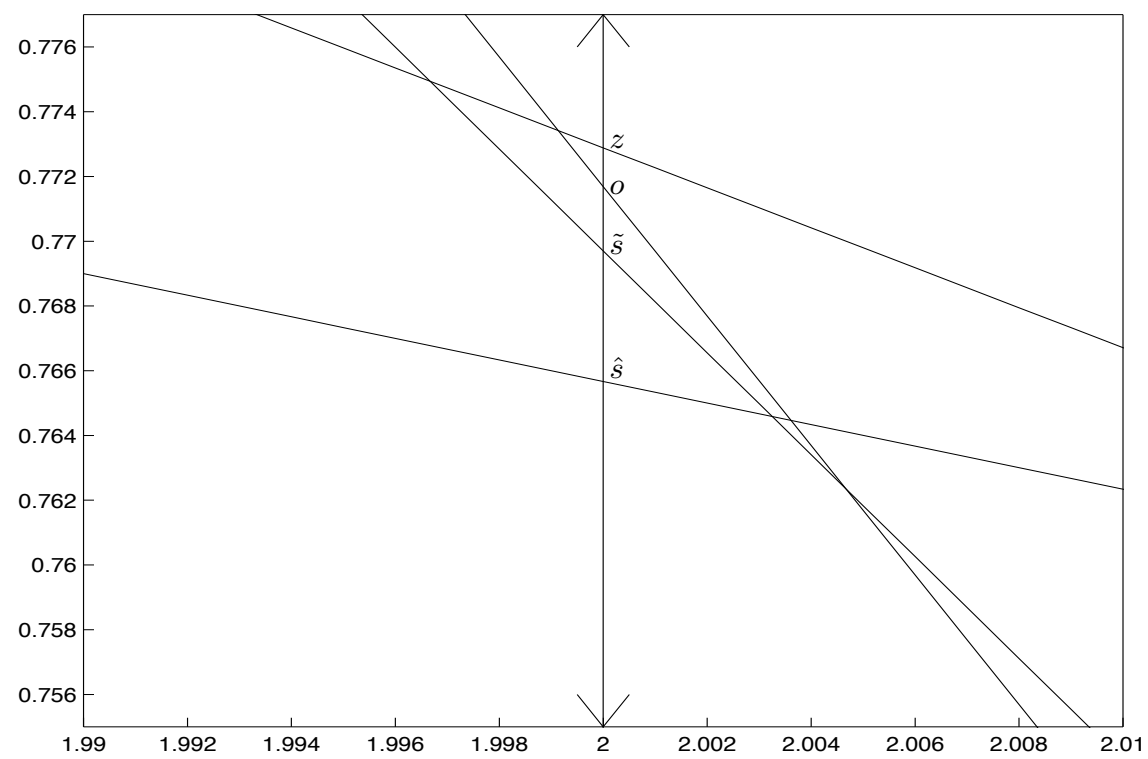

Fig. 2. Zoom of the rectangle in 1

assets denominated in the second commodity. The vertical line in figure 2 indicates the possible direction of endowment redistributions. With reference to $s=0$, in the situation $z$ the first agent has received a transfer of the second commodity but his utility decreases. In the situations $\hat{s}, \tilde{s}$ the first agent has donated some of the second commodity and with reference to $s=0$ his utility decreases. While in $\hat{s}$ it falls to the lowest of the four values, in $\tilde{s}$ it obtains a value between the utility in $s=0$ and $s=z$. Hence for these transfers the strong transfer paradox occurs and by application of our main result there exists a sunspot equilibrium with spot market endowments given by the intersection of the budget lines $\hat{s}, z$, and $\tilde{s}$ with the vertical line through the point $(2,0)$, while the selected equilibrium in reference economy has the budget line 0 . 
Although this sunspot equilibrium lies in a neighborhood of a randomization equilibrium it is itself not a randomization equilibrium because all spot market endowments differ.

This example exploits the multiplicity of equilibria of the economic fundamentals in the sense that in the neighborhood of the endowment distribution leading to multiple equilibria the budget lines have various slopes that are not ordered as the utility levels resulting in the ex post spot market equilibria (see figures 1 and 2). This property could however also occur with a unique equilibrium for the economic fundamentals. Imagine for example that the area in the rectangle of figure 1 would lie outside the Edgeworth-Box. Then keeping the line along which the transfers are defined inside the Edgeworth-Box the same construction could be done. Unfortunately, we could however give no simple utility functions as in Example 15.B.2 in [30] which would lead to this feature.

Our final example shows that sunspot equilibria do exists even if the strong uniqueness property holds. This example follows the construction of [19]. It uses Cobb-Douglas utility functions and sun-dependent assets. As compared to [19] the dimension of the example has been increased as we now use three agents, three commodities, three assets and four states.

Proposition 2. With sun-dependent assets, and under assumptions 1 and 2, the strong uniqueness assumption does not rule out the existence of sunspot equilibria.

\section{Proof}

There are three commodities and three Cobb-Douglas-consumers with utility functions:

$$
u^{i}\left(x^{i}\right)=\exp \sum_{l=1}^{3} \alpha_{l}^{i} \ln \left(x_{l}^{i}\right)
$$

The utility parameters are ${ }^{17}$ :

$$
\begin{aligned}
& \alpha^{1}=\left(\begin{array}{lll}
0.4072, & 0.5112, & 0.0816
\end{array}\right), \\
& \alpha^{2}=\left(\begin{array}{lll}
0.0269, & 0.9301, & 0.0430
\end{array}\right), \\
& \alpha^{3}=\left(\begin{array}{lll}
0.0088, & 0.0019, & 0.9892
\end{array}\right) .
\end{aligned}
$$

Note that utility functions satisfy assumption 1 and that they are linearly homogenous. Initial endowments are:

$$
\begin{aligned}
& \omega^{1}=\left(\begin{array}{lll}
0.1093, & 0.9045, & 0.1008
\end{array}\right), \\
& \omega^{2}=\left(\begin{array}{lll}
0.0715, & 0.0672, & 0.3781
\end{array}\right), \\
& \omega^{3}=\left(\begin{array}{lll}
0.8192, & 0.0283, & 0.5211
\end{array}\right) .
\end{aligned}
$$

\footnotetext{
${ }^{17}$ All values have been rounded to 4 decimal digits. The exact values can be found at the page http://www.iew.unizh.ch/home/hens/sunspot.
} 
Suppose there are four sunspot states, $s=1,2,3,4$, which are equally likely to occur. We claim that trade in numeraire but (sun-dependent) assets can be used to arrive at the following distribution of endowments:

Because asset trade only redistributes the first good, to specify the asset structure $A \in \mathbb{R}^{3 \times 4 \times 3}$, it is sufficient to give the pay offs in the numeraire, $\operatorname{good} 3$ :

$$
A_{3}=\left(\begin{array}{rrr}
410.0959 & 726.8543 & 384.2747 \\
-19.8893 & -35.3459 & -18.6341 \\
-386.5250 & -685.5971 & -362.1722 \\
0.1150 & 0.8172 & 0.0893
\end{array}\right) \text {. }
$$

Suppose equilibrium asset prices are $q=(1.0108,1.7917,0.9472)$ and agent's asset allocation are:

$$
\theta=\left(\begin{array}{rrr}
0.9911 & -0.1125 & -0.8787 \\
-0.0544 & 0.0777 & -0.0233 \\
-0.9549 & -0.0269 & 0.9818
\end{array}\right)
$$

In this matrix rows correspond to assets and columns correspond to agents. Then the resulting allocation of good three is:

$$
A_{3} \theta=\left(\begin{array}{lll}
0.1013 & 0.3767 & 0.5220 \\
0.1032 & 0.3708 & 0.5260 \\
0.1137 & 0.3385 & 0.5477 \\
0.0851 & 0.4262 & 0.4887
\end{array}\right)
$$

where rows correspond to states and columns correspond to agents.

The induced spot market equilibria have the following price vectors, allocations, values of utilities and of marginal utilities:

$$
p=\left(\begin{array}{lll}
0.4358 & 0.9623 & 1 \\
0.4324 & 0.9518 & 1 \\
0.4141 & 0.8954 & 1 \\
0.4638 & 1.0489 & 1
\end{array}\right)
$$

We give the allocations for each agent in a matrix $x^{i}$ with rows corresponding to states and columns corresponding to goods:

$$
\begin{aligned}
& x^{1}=\left(\begin{array}{lll}
0.9525 & 0.5415 & 0.0832 \\
0.9525 & 0.5432 & 0.0825 \\
0.9527 & 0.5531 & 0.0791 \\
0.9522 & 0.5285 & 0.0885
\end{array}\right), \\
& x^{2}=\left(\begin{array}{lll}
0.0291 & 0.4567 & 0.0204 \\
0.0289 & 0.4550 & 0.0200 \\
0.0278 & 0.4449 & 0.0184 \\
0.0307 & 0.4698 & 0.0228
\end{array}\right),
\end{aligned}
$$




$$
x^{3}=\left(\begin{array}{lll}
0.0184 & 0.0018 & 0.8965 \\
0.0185 & 0.0018 & 0.8974 \\
0.0194 & 0.0020 & 0.9025 \\
0.0171 & 0.0017 & 0.8887
\end{array}\right) .
$$

The corresponding levels of indirect utility are:

$$
V=\left(\begin{array}{lll}
0.7176 & 0.4248 & 0.8651 \\
0.7183 & 0.4229 & 0.8661 \\
0.7225 & 0.4122 & 0.8714 \\
0.7123 & 0.4388 & 0.8570
\end{array}\right)
$$

Finally, the levels of marginal utility within states are:

$$
\partial_{b} v=\left(\begin{array}{ccc}
0.1121 & 0.1538 & 0.2478 \\
0.1729 & 0.1537 & 0.2478 \\
0.1075 & 0.1527 & 0.2476 \\
0.1177 & 0.1531 & 0.2480
\end{array}\right) .
$$

The resulting income transfers are:

$$
A_{3} \theta=\left(\begin{array}{rrr}
0.0004 & -0.0013 & 0.0009 \\
0.0024 & -0.0073 & 0.0049 \\
0.0129 & -0.0396 & 0.0266 \\
-0.0157 & 0.0481 & -0.0324
\end{array}\right) .
$$

Note that the transfer paradox does not occur because higher transfers are always associated with higher levels of utility. However, this raises no problem here to the existence of sunspot equilibria because higher utility levels are not always associated with lower marginal utilities!

To complete the argument we need to solve the first order conditions for asset demand

$$
\sum_{s} h^{\prime i}\left(v^{i}(s)\right) \partial_{b} v^{i}(s) r^{i}(s)=0, i=1,2,
$$

by appropriate choice of the degree of the agents' risk aversion:

$$
h^{\prime}=\left(\begin{array}{lll}
0.6414 & 0.6519 & 0.9985 \\
0.9980 & 0.6583 & 0.9985 \\
0.6513 & 0.6930 & 0.9983 \\
0.6282 & 0.5979 & 0.9986
\end{array}\right) .
$$

Note that agents are risk averse since smaller values of $h^{\prime i}(s)$ are associated with higher values of utility. However, the degree of risk aversion changes in a non-trivial way with the level of utility. Those changes in risk aversion are know to be very important for asset demand. See the related papers by $[20,21,15]$ and also the monograph by $[10]$ for an extensive study. 


\section{Conclusion}

Throughout the paper we restricted attention to economies with additively separable utility functions since those functions are commonly used when applying general equilibrium models. We showed that for the case of two agents (countries) the existence of sunspot equilibria is equivalent to the occurrence of the transfer paradox. This equivalence enabled us to show that sunspots cannot matter if the economy has a unique equilibrium for all distributions of endowments induced by asset trade or if the initial economy has a unique spot market equilibrium and there are only two commodities. Then we gave an explicit example to show that with more than two commodities it is actually possible to trade from an endowment distribution leading to a unique equilibrium towards one for which there are multiple equilibria. Moreover, we gave two examples showing that sunspot equilibria need not result from multiplicity of spot market equilibria. In one of those examples, however, there are multiple equilibria at the initial distribution of endowments. In the other example there are sunspot equilibria even though the economy has a unique equilibrium for all distributions of endowments induced by asset trade. This latter example is possible because with more than two agents the existence of sunspot equilibria is no longer tied to the occurrence of the transfer paradox.

We hope that this paper has clarified the relation between the existence of sunspot equilibria and the uniqueness of spot market equilibria for economies with intrinsically complete asset markets. Moreover, further exploring the connection between sunspot equilibria and the transfer paradox, future research might also show interesting new results in related settings. For example one could try to get new insights for the sunspot literature by exploring the results on the transfer paradox in the overlapping generations model ([13]). And similarly there might be new results in storage analyzing economies with transaction costs respectively tariffs $([26])$.

\section{References}

1. Aumann R (1974) Subjectivity and Correlation in Randomized Strategies. Journal of Mathematical Economics 1(1):67-96

2. Balasko Y (1978) The Transfer Paradox and the Theory of Regular Economies. International Economic Review 19(3):687-694

3. Barnett RC, Fisher EO'N (2002) Comment on: "Do Sunpots Matter When Spot Market Equilibria Are Unique?" Econometrica 70:393-396

4. Cass D (1989) Sunspots and Incomplete Financial Markets: The Leading Example. In: Feiwell G (ed) The Economics of Imperfect Competition and Employment: Joan Robinson and Beyond. MacMillan; London

5. Cass D, Shell K (1983) Do sunsposts matter? Journal of Political Economy 91:193-227

6. Chichilniski G (1980) Basic Goods, the Effect of Aid and the International Economic Order. Journal of Development Economics 7(4):505-519 
7. Chichilniski G (1983) The Transfer Problem with Three Goods once again: Characterization, Uniqueness and Stability. Journal of Development Economics $13: 237-248$

8. Debreu G (1970) Economies with a Finite Set of Equilibria. Econometrica 38:603-615

9. Dierker E (1972) Two Remarks on the Number of Equilibria of an Economy. Econometrica 40:951-953

10. Eeckhoudt L, Gollier C (1996) Risk: Evaluation, Management and Sharing. Harvester Wheatsheaf; New York, London, Toronto

11. Eisenberg B (1961) Aggregation of Utility Functions. Management Science $7: 337-350$

12. Forges F, Peck J (1995) Correlated Equilibria and Sunspot Equilibria. Economic Theory 5(1):33-50

13. Galor O, Polemarchakis HM (1987) Intertemporal Equilibrium and The Transfer Paradox. Review of Economic Studies 54(1):147-156

14. Geanakoplos J, Heal G (1983) A Geometric Explanation of the Transfer Paradox in a Stable Economy. Journal of Development Economics 13:223-236

15. Gollier C, Pratt JW (1995) Risk Vulnerability and the Tempering Effect of Background Risk. Econometrica 64:1109-1123

16. Gottardi P, Kajii A (1999) The Structure of Sunspot Equilibria: The Role of Multiplicity. Review of Economic Studies 66:713-732

17. Guesnerie R, Laffont JJ (1988) Notes on Sunspot Equilibria in Finite Economies. In: Volume en l'Honneur d'Edmont Malinvaud,Economica-EHESS (English Version) MIT Press; Cambridge MA

18. Hara C, Segal I, Tadelis S (1997) Solutions Manual for Microeconomic Theory by Mas-Colell, Whinston and Green. Oxford University Press

19. Hens T (2000) Do Sunpots Matter When Spot Market Equilibria Are Unique? Econometrica 68:435-441

20. Hens T, Schmedders K, Voß B (1999) On Multiplicity of Competitive Equilibria when Financial Markets Are Incomplete. In: Herings PJJ, van der Laan G, Talman AJJ (eds) The Theory of Markets. North-Holland; Amsterdam

21. Hens T, Laitenberger J, Löffler A (2002) Two Remarks on the Uniqueness of Equilibria in the CAPM. Journal of Mathematical Economics 37(2):123-133

22. Jeanne O (1997) Are currency crises self-fulfilling? A test. Journal of International Economics 43:263-286

23. Jeanne O, Masson P (2000) Currency crises, sunspots and Markov-switching regimes. Journal of International Economics 50:327-350

24. Kehoe TJ (1985) Multiplicity of Equilibria and Comparative Statics. The Quarterly Journal of Economics 100:119-147

25. Kehoe TJ (1991) Computation and Multiplicity of Equilibria. In: Hildenbrand W, Sonnenschein HF (eds) Handbook of Mathematical Economics Vol. 4 : Chapter 38. North-Holland; Amsterdam

26. Lahiri S, Raimondos P (1995) Welfare Effects of Aid under Quantitative Trade Restrictions. Journal of International Economics 39(3-4):297-315

27. Leontief W (1936) Note on the Pure Theory of Transfer. Explorations in Economics, Taussig Festschrift, Vol. 84-92 New York

28. Magill M, Quinzii M (1996) Theory of Incomplete Markets. MIT Press; Cambridge MA 
29. Mas-Colell A (1992) Three Observations on Sunspots and Asset Redundandancy. In: Dasgupta P, Gale D, Hart O, Maskin F (eds) Economic Analysis of Markets and Games: Essays in Honor of Frank Hahn. MIT Press; Cambridge MA

30. Mas-Colell A, Whinston MD, Green JR (1995) Microeconomic Theory. Oxford University Press

31. Obstfeld M (1994) The Logic of Currency crises. Cahier Economiques et Monetaires (Banque de France) 43:189-213

32. Obstfeld M (1996) Models of Currency Crises with Self-Fulfilling Features. European Economic Review 40:1037-1047

33. Pilgrim B (2000) A Brief Note on Mas-Colell's Observations on Sunspots. Discussion Paper No. 450, University of Bielefeld, Germany

34. Rao M (1992) On the advantageous reallocation paradox. Social Choice and Welfare 9:131-139

35. Shoven JB, Whalley J (1995) Applying General Equilibrium. Cambridge University Press

36. Trannoy A (1986) Paradoxe Global des Transfers et Multiplicité des Equilibres: Deux Résultats. Annales D'Economie et de Statistique 4:53-61 